\title{
On the Near-Field Shaping and Focusing Capability of a Radial Line Slot Array
}

\author{
Mauro Ettorre, Member, IEEE, Massimiliano Casaletti, Member, IEEE, Guido Valerio, Member, IEEE, Ronan \\ Sauleau, Senior Member, IEEE, Laurent Le Coq, Santi C. Pavone, Student Member, IEEE, and Matteo \\ Albani, Senior Member, IEEE
}

\begin{abstract}
We describe the design of a radial line slot array antenna with a shaped and focused near field. The antenna is designed in such a way to control the side lobe level and beamwidth of the normal component of the electric field with respect to the radiating aperture. The design procedure consists of two steps. In the first step the requirements on the nearfield pattern are provided over a focusing plane at a given distance from the radiating aperture. A set theoretic approach is then used to derive the aperture field distribution fitting the requirements over the near field. In the second step, the aperture field distribution is synthesized by accurately placing and sizing the slots of the antenna. The spillover efficiency is maximized during the design process. The antenna is centrally fed by a simple coaxial probe. The antenna design is validated by a prototype and measurements at $12.5 \mathrm{GHz}$.
\end{abstract}

Index Terms - Near field, focusing, radial line slot array, RLSA, near-field shaping.

\section{INTRODUCTION}

$\mathbf{R}$ ADIAL line slot array (RLSA) antennas have been widely investigated in literature for far-field applications [1]-[4]. RLSAs are attractive antenna solutions thanks to their compactness, planarity and high efficiency. Such features are also extremely appealing for several near-field applications such as near-field probing, radiometry, non-invasive medical imaging, etc. [5]-[11]. The design techniques used for focusing and shaping the electromagnetic field in the near-field or Fresnel zone of an antenna stem from the seminal works in [12]-[14]. In these works, large (in terms of wavelength) and linearly-polarized apertures are considered and an equivalence between their near and far fields is derived. The considered near fields are either transversal or longitudinal to the axis of a defined focal plane parallel to the focusing aperture. In brief, it is shown that the fields in the Fresnel and far-field zones present the same properties if a quadratic phase taper

Manuscript received ; revised DATE.

The authors would like to thank the European Science Foundation (NEWFOCUS project), Rennes Métropole (AIS project), and IETR (Projet Prospective Scientique IETR).

M. Ettorre, Guido Valerio, R. Sauleau, and L. Le Coq are with the Institut d'Electronique et de Télécommunications de Rennes (IETR), UMR CNRS 6164, Université de Rennes 1, 35042 Rennes Cedex, France. E-mail: mauro.ettorre@univ-rennes1.fr.

M. Casaletti is with the Laboratoire d'Electronique et Electromagnétisme (L2E), Pierre and Marie Curie University - Paris 6, 75005 Paris, France. He was previously with IETR at the time of submission of the present paper. E-mail: massimiliano.casaletti@upmc.fr

S. C. Pavone and M. Albani are with the Dipartimento di Ingegneria dell'Informazione Universitá degli Studi di Siena, Via Roma 56, 53100, Siena, Italy. E-mail: matteo.albani@ing.unisi.it

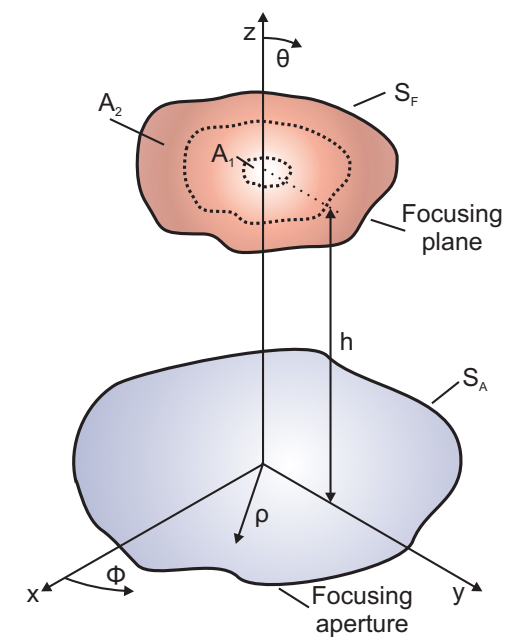

Fig. 1. Geometry of the problem with two generic surfaces defined on two parallel planes orthogonal to the $z$-axis. The focusing aperture $S_{A}$ is located at $z=0$. The focusing plane $S_{F}$ is at $z=h$. On the focusing plane the surfaces $A_{1}$ and $A_{2}$ are used in the first step of the design procedure for defining the field constraints.

is imposed to the tangential field distribution of the focusing aperture. Therefore, the optimization/shaping of the fields in the Fresnel zone is generally done by using classical farfield techniques. This is strictly valid as long as the focal plane is placed at distance larger than one aperture size and generally beyond the near-field or reactive zone of an antenna. In addition, there is no control or design flexibility on the aperture and spillover efficiency, polarization and size of the system. Besides, complicated and lossy beam forming networks are used to practically implement the required aperture field distribution introducing inevitable distortions [5]-[7].

In this paper the design of a RLSA antenna focusing its main beam in the near field is proposed. A novel design procedure is adopted for shaping the electromagnetic field radiated by the antenna without any limitation on the location of the focusing plane and polarization as in previous works [5]-[11]. In particular the possibility to control and shape the normal component of the electric field radiated by the antenna is considered as in [15]-[20]. In addition, the antenna is centrally fed by a coaxial probe avoiding lossy and cumbersome feeding networks [6], [7], [17]. The design methodology is divided in two main steps: (1) derivation of the aperture field generating the required focal pattern; (2) automatic design of the RLSA with the required aperture field distribution. 
In the first step, the required pattern or field distribution is provided based on the particular application or needs. Here, we consider the possibility to control the side lobe level and beamwidth of the normal component of the electric field of the focused beam within the near field of the structure. A set theoretic approach and alternate projection method [21] is used to derive the aperture field distribution generating the required pattern.

In the second step and in contrast to previous works [5][11], the optimization tool is linked to an in-house Method of Moment (MoM) [22]-[27] for deriving the RLSA structure having the required aperture profile. The automatic design and optimization scheme proposed in [4] is generalized for nearfield applications. The required aperture field is achieved by properly designing the slot dimensions and locations across the radiating aperture. Finally, a prototype has been manufactured and tested at the operating frequency of $f_{0}=12.5 \mathrm{GHz}$

The paper is organized as follows. Sec. II introduces the first step of the design procedure where the required aperture field distribution of the near-field focusing antenna is derived. In Sec. III, the second step of the design procedure is presented together with the RLSA geometry. The complete tool is used in Sec. IV for designing a prototype able to focus its main beam at a distance equal to half the antenna size. Details on the convergence, accuracy of the design and coaxial feeding transition are also provided. The prototype is validated by nearfield measurements of the normal component of the electric field at different frequencies and at various planes parallel to the antenna aperture. Finally, conclusions are drawn in Sec. V.

\section{Derivation of the Aperture Field Distribution}

The geometry of the problem is shown in Fig. 1. An aperture of size $S_{A}$ is located at $z=0$ and is radiating in free space. The required pattern is defined over a focusing plane $\left(S_{F}\right)$ located at a distance $z=h$ and parallel to the radiating aperture. In the following discussion, transverse magnetic (TM) modes with respect to the $z$-direction will only be considered [15], [16]. However, the proposed procedure can be extended to transverse electric (TE) modes or any combination of modes.

The goal of any synthesis procedure is to derive the aperture field distribution that generates the required pattern. A priori, such a pattern may also not be a physical solution of the problem at hand. The technique adopted here is based on a set theoretic approach and alternate projection method [21]. In these approaches, each requirement or relevant information (fabrication tolerances, synthesis error, etc.) is expressed as a set of constraints for the possible solution. The solution of the problem will belong to the intersection of all these sets of constraints or will be the closest one, according to a provided measure criterion. Circularly- and linearly-polarized fields can be considered as well as single components of the field. In the present case we will focus our attention on the possibility to control the beamwidth and sidelobe level of the $z$-component of electric field $\left(E_{z}\right)$ in the focusing plane (see Fig. 1). In addition $\phi$ invariance is assumed for the radiated field. The requirements define a mask for the near field in the focusing plane as:

$$
\text { Mask }= \begin{cases}\frac{\left|E_{z}(x, y, h)\right|}{\max \left(\left|E_{z}(x, y, h)\right|\right)}>c_{1} & \text { in } A_{1}, \\ \frac{\left|E_{z}(x, y, h)\right|}{\max \left(\left|E_{z}(x, y, h)\right|\right)}<c_{2} & \text { in } A_{2},\end{cases}
$$

where $0<c_{2}<c_{1}<1$ are fixed limits for the normalized module of $E_{z}$ in some space domains $A_{1}$ and $A_{2}$ over the focusing plane, respectively (see Fig. 1). The function $\max \left(\left|E_{z}\right|\right)$ indicates the maximum value of the $z$-component of the electric field in the focusing plane. For clarity, in the following the normalized $E_{z}$ component of the electric field in the focusing plane is indicated as:

$$
\overline{E_{z}}(x, y, h)=\frac{E_{z}(x, y, h)}{\max \left(\left|E_{z}(x, y, h)\right|\right)} .
$$

Note that the mask also defines a reference solution Ref equal to $c_{1}$ and $c_{2}$ in the two domains $A_{1}$ and $A_{2}$, respectively. The size of the focusing aperture $\left(S_{A}\right)$ is another constraint of the optimization procedure.

Once the constraints of the problem are defined, an iterative procedure based on an alternative projection method is used to derive the aperture field distribution [21]. To this end an arbitrary complex field distribution $E_{z}$ is defined at beginning over the focusing aperture: $E_{z}^{(n)}(x, y, 0)$. The apex $n$ indicates the iteration step of the procedure. The field is defined only over the surface $S_{A}$ and it is assumed zero outside this region. A uniform field distribution may be assumed as guess distribution (step $n=1$ ). The Fourier transform (FT) of the aperture field distribution is then derived:

$$
{\widetilde{E_{z}}}^{(n)}\left(k_{x}, k_{y}, 0\right)=\iint_{S_{A}} E_{z}^{(n)}(x, y, 0) e^{j\left(k_{x} x+k_{y} y\right)} d x d y,
$$

with $k_{x}$ and $k_{y}$ the spectral frequencies extending over the entire frequency spectrum $\left(-\infty<k_{x}, k_{y}<+\infty\right)$. The electric field $E_{z}(x, y, h)$ over the focusing plane can be calculated as [28]:

$$
\begin{aligned}
E_{z}^{(n)}(x, y, h)= & \frac{1}{4 \pi^{2}} \int_{-\infty}^{+\infty} \int_{-\infty}^{+\infty} \widetilde{E_{z}(n)}\left(k_{x}, k_{y}, 0\right) e^{-j k_{z} h} \\
& \times e^{-j\left(k_{x} x+k_{y} y\right)} d k_{x} d k_{y}
\end{aligned}
$$

with $k_{z}=\sqrt{k^{2}-k_{x}^{2}-k_{y}^{2}}$ and $k$ the propagation constant in free space. Therefore, for the next step $(n+1)$, the module of the derived electric field is modified accordingly to the defined mask as:

$$
\left|E_{z}^{(n+1)}(x, y, h)\right|=\left\{\begin{array}{l}
c_{1} \max \left(\left|E_{z}^{(n)}\right|\right) \text { if }\left|{\overline{E_{z}}}^{(n)}\right|<c_{1} \text { in } A_{1}, \\
c_{2} \max \left(\left|E_{z}^{(n)}\right|\right) \text { if }\left|{\overline{E_{z}}}^{(n)}\right|>c_{2} \text { in } A_{2} .
\end{array}\right.
$$

Note that the phase of the electric field is not modified. In addition, at each iteration $n$ the squared error of the field distribution is derived over the focusing plane $S_{F}$ as:

$$
\Delta_{F}^{(n)}=\iint_{S_{F}}\left|\frac{\left|E_{z}^{(n)}(x, y, h)\right|}{\max \left(\left|E_{z}^{(n)}(x, y, h)\right|\right)}-\operatorname{Ref}\right|^{2} .
$$

The FT of the electric field over the focusing plane $S_{F}$ is then evaluated as in Eq.(3) and indicated as $\widetilde{E}_{z}{ }^{(n+1)}\left(k_{x}, k_{y}, h\right)$. The 


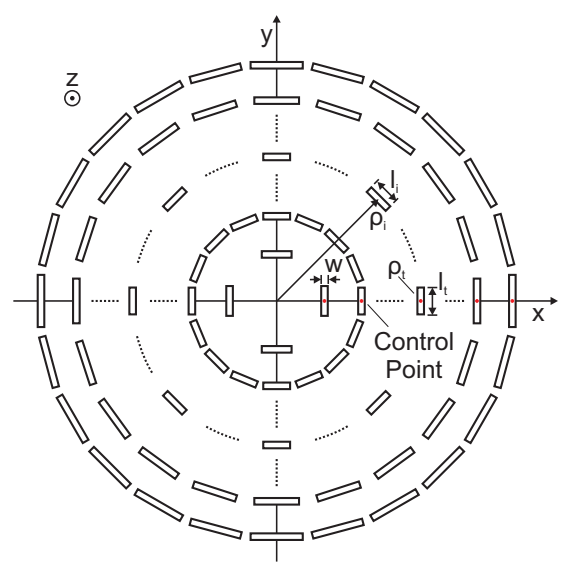

Fig. 2. Geometry of the RLSA (top view). The slots on each ring have the same length $\left(l_{i}\right)$. The width of the slots $(w)$ is constant. The slots are uniformly distributed over the rings along $\phi$. The generic control point (red dot) of the optimization procedure is indicated by the index $t$.

integration is limited to the physical size of the focusing plane $S_{F}$. The novel aperture field is obtained:

$$
\begin{aligned}
E_{z}^{(n+1)}(x, y, 0)= & \frac{1}{4 \pi^{2}} \iint_{k_{x}^{2}+k_{y}^{2} \leq k^{2}}{\widetilde{E_{z}}}^{(n+1)}\left(k_{x}, k_{y}, h\right) e^{j k_{z} h} \\
& \times e^{-j\left(k_{x} x+k_{y} y\right)} d k_{x} d k_{y}
\end{aligned}
$$

where the integral has been limited to the visible spectrum avoiding super-oscillating aperture distributions [29]. The procedure is repeated until $E_{z}$ lays within the imposed requirements in the focusing plane or the squared error $\Delta_{F}$ is lower than a certain threshold. It is worth saying that the FTs in Eqs. (4), (7) have been evaluated with a Fast Fourier Transform (FFT) algorithm speeding up their computation and the overall time convergency of the design procedure.

The present procedure provides the $z$-component of electric field $E_{z}$ over the focusing aperture [15]-[16]. However, in the next step the tangential electric field distribution is required for designing the RLSA. Thanks to the $\phi$ invariance of the radiated field, the $\rho$-component of the tangential electric field is given by:

$$
\begin{aligned}
E_{\rho}(x, y, 0)= & \frac{1}{4 \pi^{2}} \int_{-\infty}^{+\infty} \int_{-\infty}^{+\infty} \frac{k_{z}}{k_{\rho}} \widetilde{E_{z}}\left(k_{x}, k_{y}, 0\right) \\
& \times e^{-j\left(k_{x} x+k_{y} y\right)} d k_{x} d k_{y}
\end{aligned}
$$

with $k_{\rho}=\sqrt{k_{x}^{2}+k_{y}^{2}}$.

Finally, the scalar procedure presented here can be easily extended to a more general vector approach. In addition, the procedure can be directly applied to the tangential components of the electric field in place of the vertical one.

\section{Synthesis of the Aperture Field Distribution}

The design of the RLSA structure follows the same steps outlined in [4]. The derived aperture field distribution in Eq.(8) corresponds to an equivalent aperture magnetic current distribution: $\mathbf{M}_{\mathbf{0}}(\rho)=M_{0}(\rho) \widehat{\phi}=-E_{\rho}(\rho) \widehat{\phi}$. Note that the current distribution is oriented along $\phi$ and is a function only of $\rho$ due to the $\phi$-invariance of the assumed TM modes in

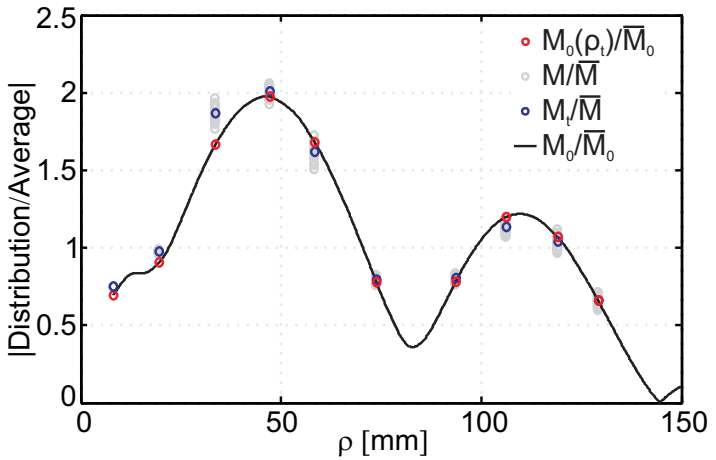

(a)

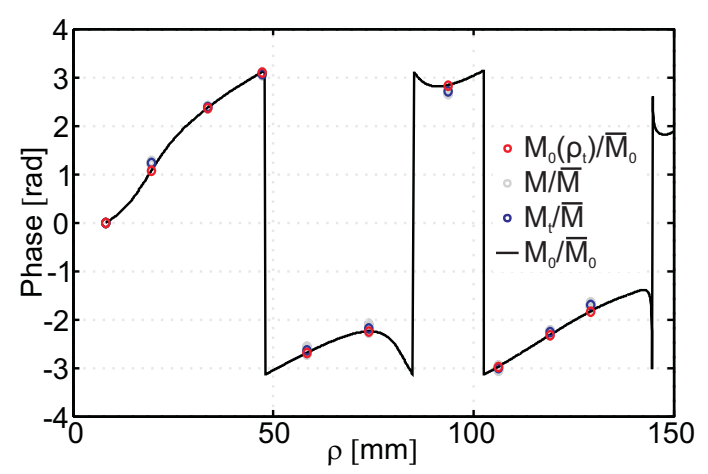

(b)

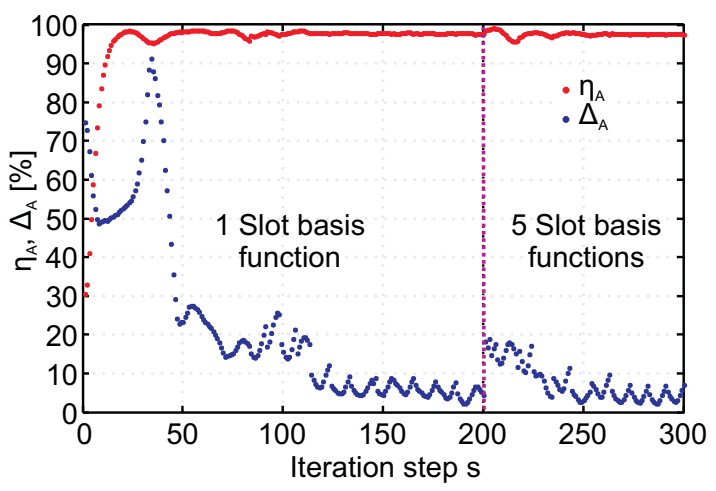

(c)

Fig. 3. (color) Ideal and synthesized magnetic dipole moment distribution provided by the two steps of the design procedure for the final RLSA antenna: (a) magnitude; (b) phase. (c) Spillover efficiency $\left(\eta_{A}\right)$ and average aperture distribution error $\left(\Delta_{A}\right)$ vs. iteration step $(s)$ with a different number of entire domain basis functions for the analysis of the RLSA with the MoM code.

the first step of the design process. The current distribution is synthesized by using the slots of the RLSA.

Each slot is equivalent to a magnetic dipole oriented along the slot length. The dipole moment is proportional to the feeding mode (outwards cylindrical wave) within the parallel plate waveguide (PPW) of the RLSA antenna and function of the position and size of the corresponding slot. The slots, and 
TABLE I

POSITION AND DIMENSIONS (in $\mathrm{mm}$ ) OF THE SLOTS IN EACH RING OF THE RLSA

\begin{tabular}{|c|c|c|c|c|c|c|c|c|c|c|}
\hline Ring number & 1 & 2 & 3 & 4 & 5 & 6 & 7 & 8 & 9 & 10 \\
\hline Length & 5.23 & 5.9 & 8.08 & 7.35 & 8.3 & 7.89 & 7.28 & 8.33 & 8.68 & 10.19 \\
\hline$\rho_{t}$ & 8.15 & 19.54 & 33.61 & 47.13 & 58.38 & 73.80 & 93.57 & 106.16 & 119.05 & 129.20 \\
\hline
\end{tabular}

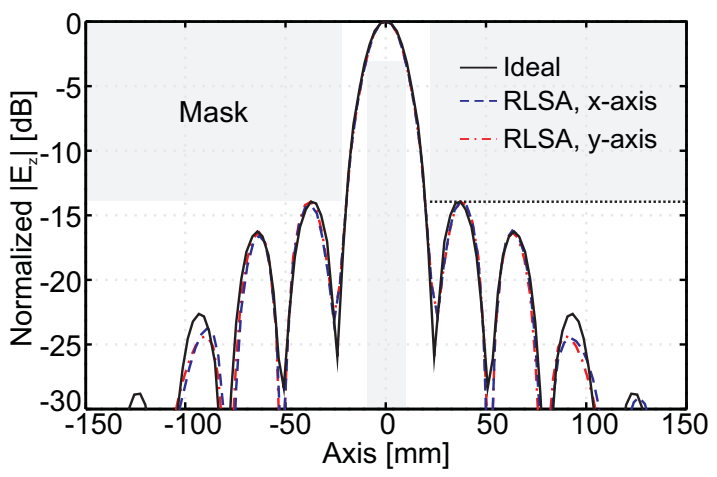

Fig. 4. (color) Normalized $E_{z}$ component of the electric field in the focusing plane $(z=150 \mathrm{~mm})$ radiated by: ideal aperture field distribution (solid line); synthesised RLSA (blue dashed line along the $x$-axis, red dash-dotted line along the $y$-axis). The reference mask is also provided for comparison.

as a consequence their equivalent magnetic dipoles, must be oriented in the same direction as the current distribution $\left(\mathbf{M}_{\mathbf{0}}\right)$ for its synthesis [4].

The resulting geometry of the RLSA antenna is shown in Fig. 2 (top view). The slots are placed in the upper plate of a PPW structure filled by a dielectric with permittivity $\epsilon_{r}$ [1], [2]. Slots of same length $\left(l_{i}\right)$ are uniformly spaced in each ring and oriented along $\phi$ thanks to $\phi$-invariance of the assumed current distribution. It is worth saying that the mutual coupling between each slot of any ring and the surrounding slots may be different and may induce small asymmetries in the radiated field. The width of the slots is constant.

For each slot of the RLSA the equivalent magnetic dipole moment $\mathbf{M}\left(\boldsymbol{\rho}_{i}\right)=M\left(\boldsymbol{\rho}_{i}\right) \widehat{\phi}$ is derived with an in-house MoM [22], [23]. The position $\left(\rho_{t}\right)$ and length $\left(l_{t}\right)$ of one representative slot per ring (control point in Fig. 2) is adjusted for synthesising the derived aperture magnetic current distribution $\mathbf{M}_{\mathbf{0}}$. This is possible thanks to the assumption of equal slots in each ring of the RLSA. In other words the magnetic dipole moment is averaged over each ring, yielding:

$$
M_{t}\left(\rho_{t}\right)=\frac{1}{N_{t}} \sum_{i=1}^{N_{t}} M\left(\boldsymbol{\rho}_{i}\right)
$$

where $N_{t}$ is the number of slot on ring $t$. For each slot or control point (see Fig. 2) a complex fitness function is defined:

$$
F_{t}=\frac{M_{t}\left(\rho_{t}\right)}{M_{0}\left(\rho_{t}\right)} \frac{\overline{M_{0}}}{\bar{M}}
$$

where $\bar{M}=\sum_{t=1}^{N_{\text {ring }}}\left|M_{t}\left(\rho_{t}\right)\right| N_{t} / N_{\text {slot }}$ and $\overline{M_{0}}=$ $\sum_{t=1}^{N_{\text {ring }}}\left|M_{0}\left(\rho_{t}\right)\right| N_{t} / N_{\text {slot }}$ are the realized and target average magnetic dipole moment amplitude, with $N_{\text {slot }}$ and $N_{\text {ring }}$ the total number of slots and rings. The phase $\left(\angle F_{t}\right)$ and amplitude $\left(\left|F_{t}\right|\right)$ of the fitness function represent the error between the target and actual magnetic dipole moment distribution over the

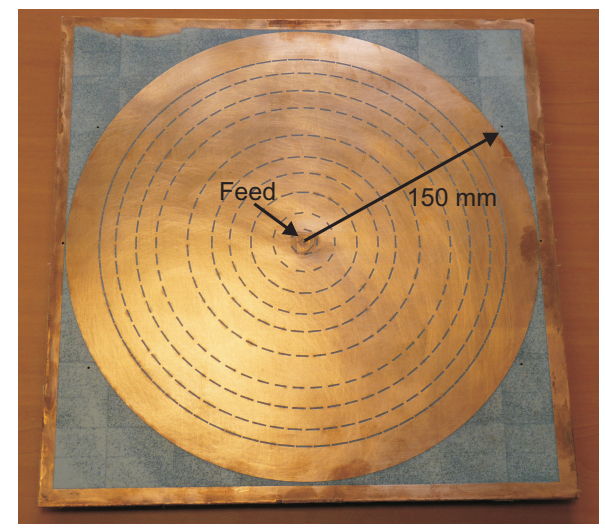

Fig. 5. Final prototype. The slots have been etched by laser ablation.

TABLE II

GEOMETRICAL DIMENSIONS (in $\mathrm{mm}$ ) FOR THE COAXIAL TRANSITION OF THE PROTOTYPE

\begin{tabular}{|c|c|c|c|c|}
\hline$D_{1}$ & $D_{2}$ & $D_{3}$ & $h_{1}$ & $h_{d}$ \\
\hline 1.28 & 2.34 & 7.68 & 1.58 & 3.175 \\
\hline
\end{tabular}

aperture. The fitness function is used to modify the position $\left(\rho_{t}\right)$ and length of the slots $\left(l_{s}\right)$ within an optimization loop. At each iteration $(s)$ of the optimization process, the inhouse MoM is used to evaluate the slots' magnetic dipole moment distribution. The fitting function $F_{t}^{(s)}$ is then derived. In addition, the spillover efficiency of the antenna is also calculated:

$$
\eta_{A}^{(s)}=\frac{P_{\mathrm{acc}}-P_{\mathrm{res}}}{P_{\mathrm{acc}}}
$$

with $P_{\text {acc }}$ and $P_{\text {res }}$ the power accepted by the antenna and the residual power trapped in the PPW, respectively. The spillover efficiency is maximized during the optimization. The position and length of the slots are updated accordingly as:

$$
\begin{aligned}
\rho_{t}^{(s+1)} & =\rho_{t}^{(s)}+\xi_{p} \angle F_{t}^{(s)} / k_{d}, \\
l_{t}^{(s+1)} & =l_{t}^{(s)}+\xi_{q}\left(1-\left|F_{t}^{(s)}\right| \sqrt{\eta_{A}^{(s)}}\right) l_{t}^{(s)},
\end{aligned}
$$

where $k_{d}=k \sqrt{\epsilon_{r}}$ is the wavenumber in the dielectric filling the PPW, and $0<\xi_{p}, \xi_{q}<1$ are damping factors used in the optimization loop [4]. Note that the number of slots in each ring $\left(N_{t}\right)$ is calculated as:

$$
N_{t}^{(s+1)}=\left\lfloor\frac{2 \pi \rho_{t}^{(s+1)}}{l_{\max }}\right\rfloor
$$

where $l_{\max }$ is the upper limit for the slot length which is set to the resonant slot length [4]. The notation \lfloor\rfloor indicates the floor function. The convergence criteria of the optimization loop are based on the spillover efficiency, and the average 


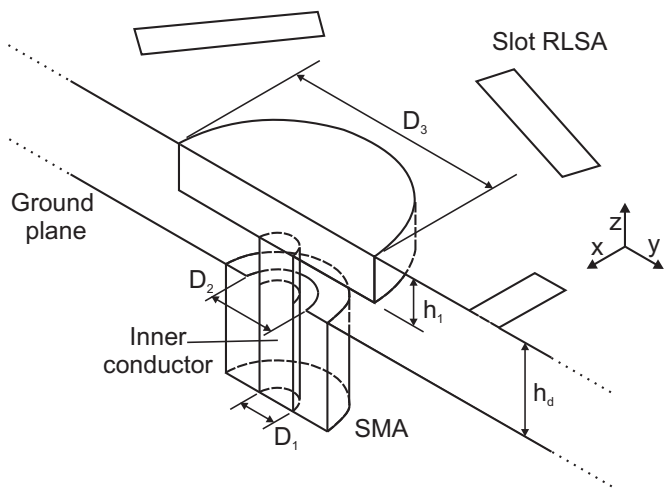

Fig. 6. Coaxial transition. The metallic disc of diameter $D_{3}$ and thickness $h_{1}$ is inserted in the substrate of the RLSA structure and attached to the inner conductor of the SMA connector. The SMA connector is placed in the back side of the antenna.

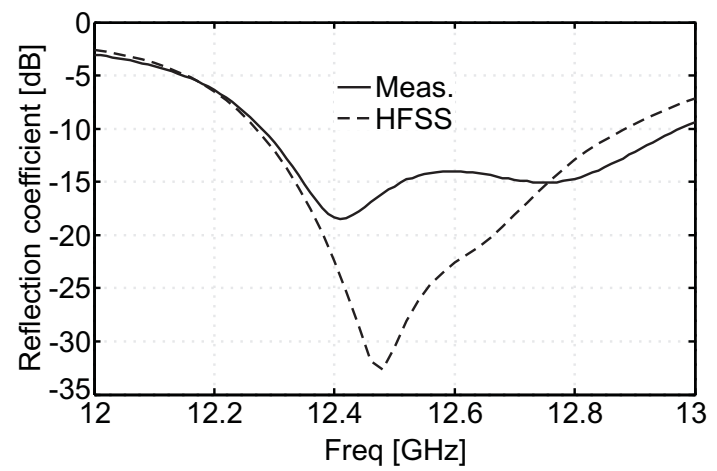

Fig. 7. Simulated and measured input reflection coefficient. The simulation results have been obtained by the general purpose software HFSS [30].

aperture distribution error given by:

$$
\Delta_{A}=\frac{1}{N_{\text {slot }}} \sum_{t=1}^{N_{\text {ring }}}\left|\frac{M_{t}\left(\rho_{t}\right)}{\bar{M}}-\frac{M_{0}\left(\rho_{t}\right)}{\overline{M_{0}}}\right| N_{t} .
$$

Note that the proposed optimization loop adopts a projection method as in the first step of the design procedure [21]. In addition, the procedure proposed in this section can be extended to more general configurations with different slot patterns for the RLSA structure, as shown in [4] for far-field applications using RLSA antennas as those in [1]-[3].

\section{PROTOTyPE AND EXPERIMENTAL RESUlts}

The procedure presented in the previous sections is used to design a RLSA with a shaped $z$-component of the electric field $\left(E_{z}\right)$ (see Fig. 1). The operating frequency is chosen equal to $f_{0}=12.5 \mathrm{GHz}$. The physical constraints for the first step of the design procedure in Sec. II are: $S_{A}<300 \mathrm{~mm}^{2} ; h=$ $150 \mathrm{~mm}$. The antenna focuses $E_{z}$ at a distance equal to half the antenna size. In addition the following mask is imposed on the normalized $E_{z}$ profile over the focusing plane in terms of side lobe level (SLL) and beamwidth:

$$
\text { Mask }= \begin{cases}\frac{\left|E_{z}\right|}{\max \left(\left|E_{z}\right|\right)}>-3 \mathrm{~dB} & \text { for } \rho<10 \mathrm{~mm} \\ \frac{\left|E_{z}\right|}{\max \left(\left|E_{z}\right|\right)}<-14 \mathrm{~dB} & \text { for } \rho>20 \mathrm{~mm}\end{cases}
$$

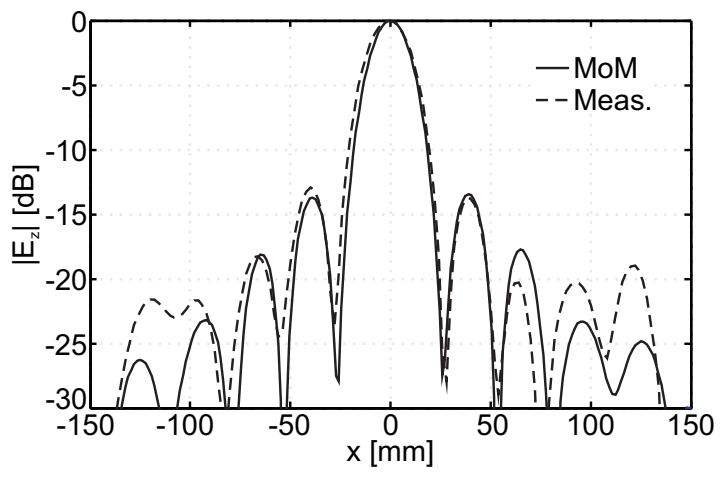

(a)

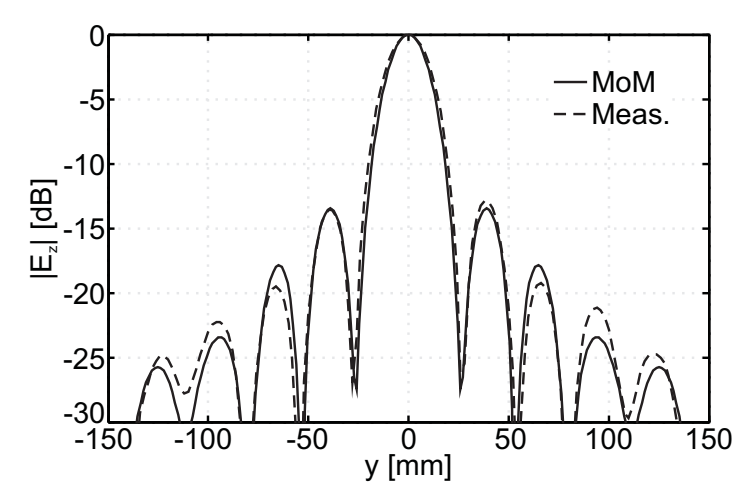

(b)

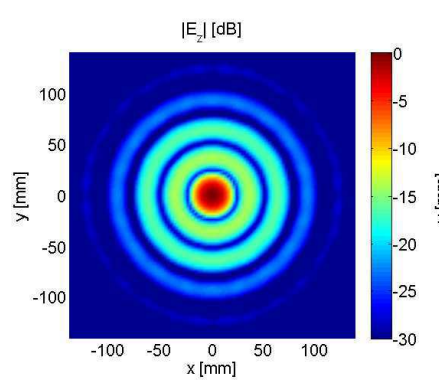

(c)

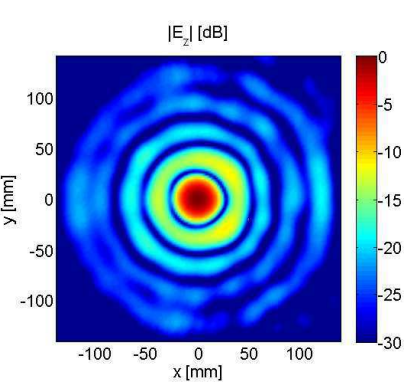

(d)
Fig. 8. (color) Normalized $E_{z}$ component of the electric field at $f_{0}$ on the focusing plane. (a) Comparison between measured and MoM results along the $x$-axis. (b) Comparison between measured and MoM results along the $y$-axis. (c) 2D MoM electric field plot. (d) 2D measured electric field plot.

Note that this mask is chosen as an example. However, other requirements may be defined based on the considered target application.

For the second step (Sec. III), the convergence criteria are the followings: $\eta_{A}>95 \% ; \Delta_{A}<3 \%$. The RLSA antenna is made by a PPW of height $h_{d}=3.175 \mathrm{~mm}$ and filled by a dielectric with permittivity $\epsilon_{r}=2.17$. The final positions and lenghts of the slots are given in Table I. The diameter of the antenna is smaller than $300 \mathrm{~mm}$. The final RLSA structure has a spillover efficiency and average aperture distribution error 


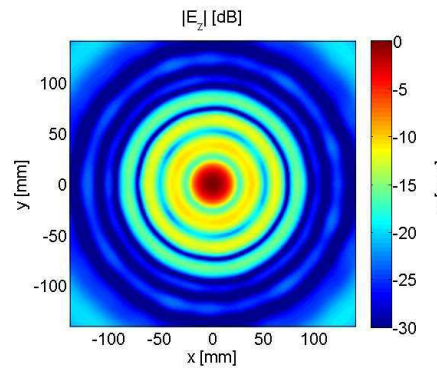

(a)

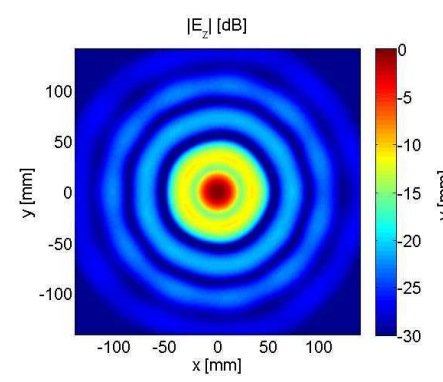

(c)

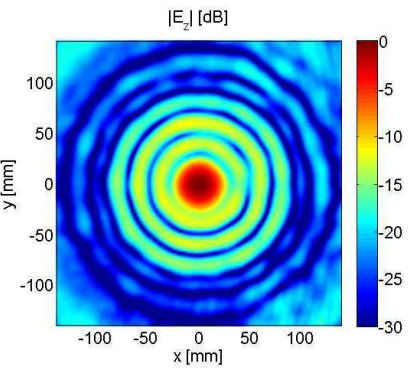

(b)

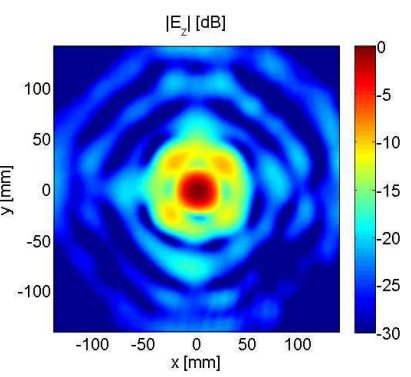

(d)

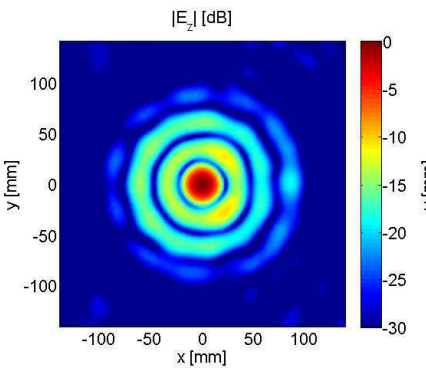

(a)

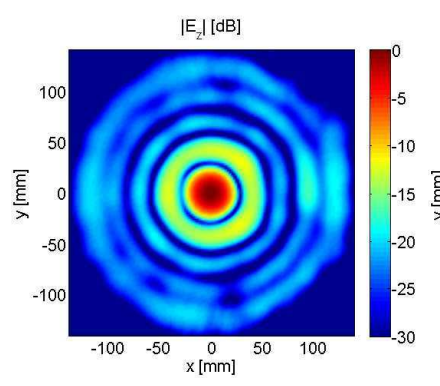

(c)

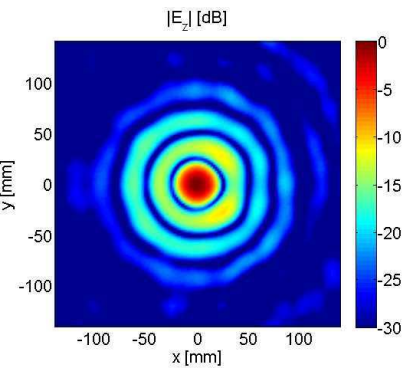

(b)

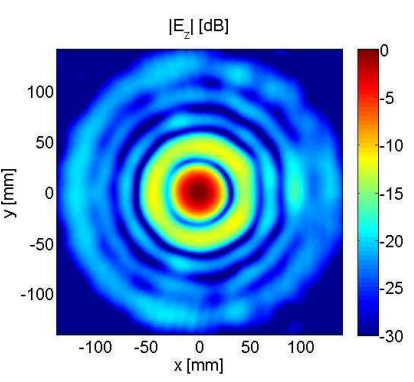

(d)
Fig. 9. (color) Normalized $E_{z}$ component of the electric field at $f_{0}$ on two different planes parallel to the RLSA aperture. (a) 2D MoM electric field plot at $z=100 \mathrm{~mm}$. (b) 2D measured electric field plot at $z=100 \mathrm{~mm}$. (c) 2D MoM electric field plot at $z=200 \mathrm{~mm}$. (d) 2D measured electric field plot at $z=200 \mathrm{~mm}$.

of $97.8 \%$ and $2.2 \%$, respectively. The ideal and synthesized magnetic dipole moment distribution are shown and compared in Fig. 3 in amplitude and phase. The blue and grey circles indicate the averaged magnetic dipole moment at the control point $\left(M_{t} / \bar{M}\right)$ and at each slot in the rings of the RLSA $(M / \bar{M})$, respectively. The black line is the ideal averaged magnetic current distribution over the aperture provided by Eq.(8) $\left(M_{0} / \overline{M_{0}}\right)$. The red circles are the samples of this distribution at the location of the control point $\rho_{t}$ in each ring $\left(M_{0}\left(\rho_{t}\right) / \overline{M_{0}}\right)$. A good agreement can be appreciated between the ideal profile (black curve and red circles) and the real one (blue circles) of the RLSA antenna. In some cases, a small variation in absolute value can be noticed between the slots' magnetic dipole moment of a particular ring (grey circles in Fig. 3 (a)) and their average distribution (blue circles in Fig. 3 (a)). This is due to the different mutual coupling between each slot of each ring and the surrounding slots of the RLSA structure.

The variation of the spillover efficiency $\left(\eta_{A}\right)$ and average aperture distribution error $\left(\Delta_{A}\right)$ with respect to the iteration step $(s)$ is shown in Fig. 3. It can be noticed that $\eta_{A}$ converges faster than $\Delta_{A}$ to acceptable values. Fig. 3 also shows the effect of using a different number of entire domain basis functions in the MoM code for the analysis of the RLSA. In particular, 1 basis function is used at the beginning of Step 2 of the design procedure to speed up the optimization process. Once a solution is reached, the number of basis functions is
Fig. 10. (color) Normalized 2D measured $E_{z}$ component of the electric field on the focusing plane at various frequencies. (a) $f=12.3 \mathrm{GHz}$. (b) $f=12.4$ GHz. (c) $f=12.6 \mathrm{GHz}$. (d) $f=12.7 \mathrm{GHz}$.

increased to 5 for a higher accuracy of the MoM analysis and final results. This approach is possible thanks to the extreme efficiency in computational time of the in-house MoM [22], [23]. Note that the final RLSA has 400 slots.

The normalized $E_{z}$ component of the electric field radiated by the RLSA along the $x$ - and $y$-axis on the focusing plane is shown in Fig. 4. The field radiated by the derived ideal aperture field distribution in Eq.(8) is also shown for comparison. A small difference can be noticed in the lobes lower than $-20 \mathrm{~dB}$ but in all cases the patterns are within the imposed mask. $\phi$ invariance.

A prototype of the RLSA has been manufactured and tested at IETR. The prototype is shown in Fig. 5. The slots of the RLSA are etched by laser ablation on the upper face of a double grounded Neltec NY9217 substrate with permittivity $\epsilon_{r}=2.17$ and thickness $h_{d}=3.175 \mathrm{~mm}$. The prototype is centrally fed by a coaxial probe attached on the back side of the prototype to a SMA connector. The coaxial transition shown in Fig. 6 is used to match the antenna to the $50 \mathrm{Ohm}$ SMA connector. The transition is made by a metallic disc of diameter $D_{3}$ and thickness $h_{1}$ connected to the inner conductor of the SMA connector. The final dimensions for the coaxial transition are provided in Table II. The symbols refer to Fig. 6. The simulated and measured reflection coefficients are plotted in Fig. 7, and show good agreement.

The $E_{z}$ component of the electric field was measured above the prototype using a short electric probe attached to Besides, the field radiated by the RLSA antenna have a good 


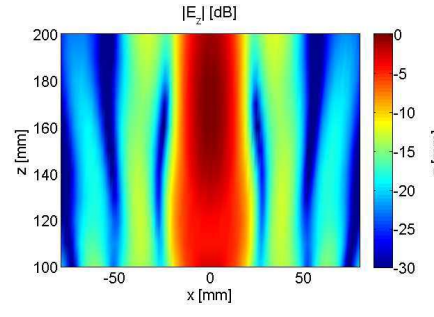

(a)

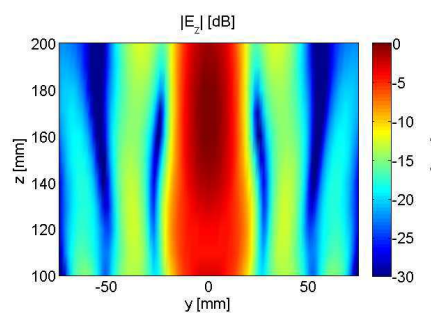

(c)

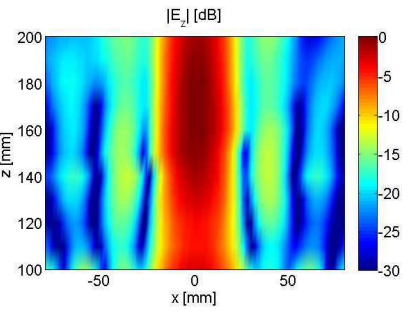

(b)

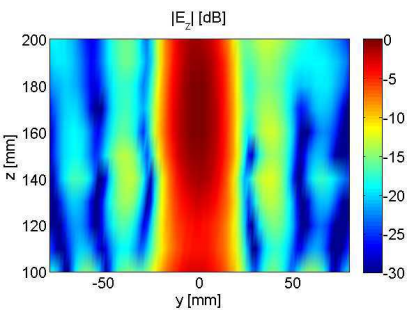

(d)
Fig. 11. (color) Normalized $E_{z}$ component of the electric field at the operating frequency. (a) 2D MoM electric field plot along the $x z$-plane. (b) 2D measured electric field plot along the $x z$-plane. (c) 2D MoM electric field plot along the $y z$-plane. (d) 2D measured electric field plot along the $y z$-plane.

an automatically controlled 2D translation stage. The short electric probe is made out of a semi-rigid coaxial cable (UT-85) by removing the external conductor and dielectric insulator. The inner conductor extends for a length of $2.5 \mathrm{~mm}$ (about $\lambda / 10$ with $\lambda$ the free space wavelength at the operating frequency) beyond the outer conducting shield. The near field was measured over an area of $150 \times 150 \mathrm{~mm}^{2}$ with a step of $2 \mathrm{~mm}$ at different distances from the RLSA structure. The normalized $E_{z}$ component at the operating frequency $f_{0}$ along the $x$ - and $y$-axis is shown in Fig. 8 (a) and (b), respectively. Good agreement is achieved between the measured and simulated results. This is also evident in the 2D plots of the fields in Fig. 8 (c) and (d). A good circular symmetry of the pattern can be appreciated. However small discrepancies between measured and simulated results can be noticed for side lobes lower than $-15 \mathrm{~dB}$ at the edge of the measured space. Such discrepancies may be attributed to the fabrication tolerances related to the etching process used for the slots of the RLSA structure.

To further validated the design, 2D plots of the normalized electric field are provided in Fig. 9 at difference distances from the RLSA: $z=100 \mathrm{~mm} ; z=200 \mathrm{~mm}$. Once again, a close agreement is observed between the simulated and measured results even outside the focusing plane. The variation of the measured field within the band $12.3-12.7 \mathrm{GHz}$ is shown in Fig. 10. It can be noticed that the field pattern is preserved within the considered band. For brevity, only the measurement results are provided. However also for these cases there was a good agrement with the simulated ones.
Finally, Fig. 11 provides the 2D plot of the normalized $E_{z}$ along the $x z$ - and $y z$-plane. The field was measured with a step of $10 \mathrm{~mm}$ in $z$. At the time of the measurements the near-field scanner did not have a translation stage for the $z$ axis. Therefore, the probe was manually adjusted along $z$. For this reason, the measured fields are normalized along $z$ using the MoM results to prevent any artefact or misleading information due to the measurement limitations. In other words, the maximum along the $z$-axis is the same for both the measured and MoM results. The focusing behaviour and good accuracy of the measured near field along $z$ can be appreciated.

\section{CONCLUSION}

In this paper, the design and measurement results of a radial line slot array antenna focusing in its near field have been presented. The possibility to control the side lobe level and beamwidth of the vertical component of the electric field has been considered. The RLSA structure is automatically generated by a novel design procedure. The input information of the design procedure are the size of the antenna and the requirements on the near-field pattern. The procedure is divided in two main steps. In the first step the aperture field distribution generating the required near field is derived. In the second step, the position and size of the slots of the RLSA are adjusted for synthesizing the derived aperture field and maximizing the spillover efficiency. An in-house fast MoM is used in the second step of the design procedure and for the analysis of the structure. General purpose software can not be used during this step due to their prohibitive computational time. In addition, the antenna can not be designed with classical near-field methodologies based on an equivalence between the near and far field of an antenna. Exhaustive 2D measurements validated the proposed approach and realized prototype. The proposed structure and design technique may find application in areas such as near-field probing and radiometry, wireless power transfer, medical imaging, and non-diffractive radiation.

\section{REFERENCES}

[1] N. Goto and M. Yamamoto, "Circularly polarized radial-line slot antennas," IECE Japan, Tech. Rep., APSO-57, Aug. 1980.

[2] M. Ando, K. Sakurai, N. Goto, K. Arimura, and Y. Ito, "A radial line slot antenna for $12 \mathrm{GHz}$ satellite TV reception," IEEE Trans. Antennas Propag., vol. 33, no. 12, pp. 1347-1353, Dec. 1985.

[3] P. W. Davis and M. E. Bialkowski, "Experimental investigations into a linearly polarized radial slot antenna for DBS TV in Australia," IEEE Trans. Antennas Propag., vol. 45, no. 7, pp. 1123-1129, Jul. 1997.

[4] M. Albani, A. Mazzinghi, and A. Freni, "Automatic design of CP-RLSA antennas," IEEE Trans. Antennas Propag., vol. 60, no. 12, pp. 55385547, Dec. 2012.

[5] A. Buffi, P. Nepa, and G. Manara, "Design criteria for near-field-focused planar arrays," IEEE Trans. Antennas Propag., vol. 14, no. 1, pp. 40-50, Feb. 2012.

[6] K. D. Stephan, J. B. Mead, D. M. Pozar, L. Wang, and J. A. Pearce, "A near field focused microstrip array for a radiometric temperature sensor," IEEE Trans. Antennas Propag., vol. 55, no. 4, pp. 1199-1203, Apr. 2007.

[7] M. Bogosanovic and A. G. Williamson, "Microstrip antenna array with a beam focused in the near-field zone for application in noncontact microwave industrial inspection," IEEE Trans. Instrum. Meas., vol. 56, no. 6, pp. 2186-2195, Dec. 2007.

[8] C. H. Durney and M. F. Iskandar, "Antennas for medical applications," Antenna Hand Book: Theory, Applications, and Design., Y. T. Lo and S. W. Lee, Eds. New York: Van Nostrand, 1988, ch. 24. 
[9] W. C. Brown, "The history of power transmission by radio waves," IEEE Trans. Microw. Theory Tech., vol. 32, no. 9, pp. 1230-1242, Sep. 1984.

[10] J. L. Gómez-Tornero, D. Blanco, E. Rajo-Iglesias, and N. Llombart, "Holographic surface leaky-wave lenses with circularly-polarized focused near-fields. Part I: concept, design and analysis theory," IEEE Trans. Antennas Propag., vol. 61, no. 7, pp. 3475-3485, Jul. 2013.

[11] D. Blanco, J. L. Gómez-Tornero, E. Rajo-Iglesias, and N. Llombart, "Holographic surface leaky-wave lenses with circularly-polarized focused near-fields. Part II: experiments and description of frequency steering of focal length," IEEE Trans. Antennas Propag., vol. 61, no. 7, pp. 3486-3494, Jul. 2013

[12] J. W. Sherman, "Properties of focused apertures in the Fresnel region," IRE Trans. Antennas Propag., vol. 10, no. 4, pp. 399-408, Jul. 1962.

[13] W. J. Graham, "Analysis and synthesis of axial field pattern of focused apertures," IEEE Trans. Antennas Propag., vol. 31, no. 4, pp. 665-668, Jul. 1983.

[14] R. C. Hansen, "Focal region characteristics of focused array antennas," IEEE Trans. Antennas Propag., vol. 33, no. 12, pp. 1328-1337, Dec. 1985.

[15] M. Ettorre and A. Grbic, "Generation of propagating Bessel beams using leaky-wave modes," IEEE Trans. Antennas Propag., vol. 60, no. 8, pp. 3605-3605, Aug. 2012.

[16] M. Ettorre, S. M. Rudolph, and A. Grbic, "Generation of propagating Bessel beams using leaky-wave modes: experimental validation," IEEE Trans. Antennas Propag., vol. 60, no. 6, pp. 2645-2653, Jun. 2012.

[17] P. Lemaitre-Auger, S. Abielmona, and C. Caloz, "Generation of Bessel beams by two-dimensional antenna arrays using sub-sampled distributions," IEEE Trans. Antennas Propag., vol. 61, no. 4, pp. 1838-1849, Apr. 2013.

[18] Y. Yasaka, N. Ishii, T. Yamamoto, M. Ando, and M. Takahashi, “ Development of a slot-excited planar microwave discharge device for uniform plasma processing," IEEE Trans. Plasma Sci., vol. 32, no. 1, pp. 101-107, Feb. 2004

[19] M. A. Salem, A. H. Kamel, and E. Niver, "Microwave Bessel beams generation using guided modes," IEEE Trans. Antennas Propag., vol. 59, no. 2, pp. 2241-2247, Jun. 2011.

[20] M. F. Imani and A. Grbic, "Generating evanescent Bessel beams using near-field plates," IEEE Trans. Antennas Propag., vol. 60, no. 7, pp. 3155-3164, Jul. 2012

[21] R. Piestun and J. Shamir, "Synthesis of three-dimensional light fields and applications," Proc. IEEE, vol. 90, no. 2, pp. 222-244, Feb. 2002.

[22] M. Casaletti, R. Sauleau, M. Ettorre, and S. Maci, "Efficient analysis of metallic and dielectric posts in parallel plate waveguide structures," IEEE Trans. Microw. Theory Tech., vol. 60, no. 10, pp. 2979-2989, Oct. 2012.

[23] M. Casaletti, G. Valerio, J. Seljan, M. Ettorre, and R. Sauleau, "A full-wave hybrid method for the analysis of multilayered SIW-based antennas," forthcoming in IEEE Trans. Antennas Propag., 2013.

[24] M. Albani, G. Lo Cono, R. Gardelli, and A. Freni, "An efficient fullwave method of moments analysis for RLSA antennas," IEEE Trans. Antennas Propag., vol. 54, no. 8, pp. 2326-2336, Aug. 2006.

[25] M. Albani, A. Mazzinghi, and A. Freni, "Asymptotic approximation of mutual admittance involved in MoM analysis of RLSA antennas," IEEE Trans. Antennas Propag., vol. 57, no. 4, pp. 1057-1063, Apr. 2009.

[26] A. Mazzinghi, A. Freni, and M. Albani, "Influence of the finite slot thickness on RLSA antenna design," IEEE Trans. Antennas Propag., vol. 58, no. 1, pp. 215-218, Jan. 2010.

[27] M. Albani, A. Mazzinghi, and A. Freni, "Rigorous MoM analysis of finite conductivity effects in RLSA antennas," IEEE Trans. Antennas Propag., vol. 59, no. 11, pp. 4023-4032, Nov. 2011.

[28] C. A. Balanis, Antenna Theory: Analysis and Design, 2nd ed., Chap. 12, John Wiley \& Sons, Inc., New York, 1997.

[29] A. Grbic and R. Merlin, "Near-field focusing plates and their design," IEEE Trans. Antennas Propag., vol. 56, no. 10, pp. 3159-3165, Oct. 2008 .

[30] Ansoft HFSS version 12.0, 1984-2010 Ansoft Corporation.

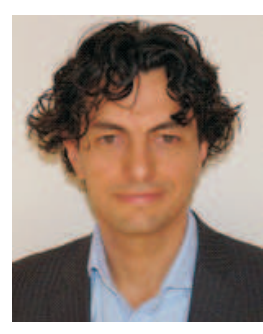

Mauro Ettorre (M'08) was born in Tricarico, Matera, Italy, in 1979. He received the laurea degree (summa cum laude) in telecommunication engineering and the Ph.D. degree in electromagnetics from the University of Siena, Italy, in 2004 and 2008 respectively.

During his Master's degree studies he spent five months at the Technical University of Denmark (DTU), Lyngby, Denmark. Part of his PhD has been developed at the Defence, Security and Safety Institute of the Netherlands Organization for Applied Scientific Research (TNO), The Hague, The Netherlands, where afterwards he worked as antenna researcher. From 2008 to 2010, he was a Postdoctoral Fellow at the Institut d'Electronique et de Télécommunications de Rennes (IETR), Université de Rennes 1, France. From 2010 to 2011, he was a visiting scholar at the Radiation Laboratory, Department of Electrical Engineering and Computer Science, University of Michigan, Ann Arbor, USA. Since October 2010, he has been appointed permanent CNRS researcher at IETR.

His research interests include the analysis and design of leaky-wave antennas, periodic structures, compact planar antennas and wireless power transmission systems.

Dr. Ettorre received the Young Antenna Engineer Prize during the 30th ESA Antenna Workshop 2008 in Noordwijk, The Netherlands.

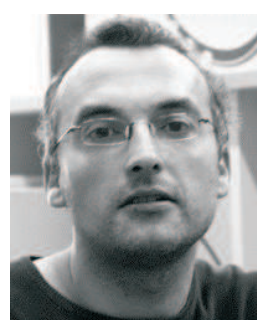

Massimiliano Casaletti was born in Siena, Italy, in 1975. He received the Laurea degree in telecommunications engineering and the $\mathrm{Ph} . \mathrm{D}$. degree in information engineering from the University of Siena, Italy, in 2003 and 2007, respectively. From September 2003 to October 2005, he was with the research center MOTHESIM, Les Plessis Robinson (Paris, FR), under EU grant RTN-AMPER (RTN: Research Training Network, AMPER: Application of Multiparameter Polarimetry). He has been Research Associate at the University of Siena, Italy from November 2006 until October 2010 and a Postdoctoral Researcher from November 2010 to August 2013 at the IETR (Institut d'Electronique et des Télécommunications de Rennes) at University of Rennes 1, Rennes, France. $\mathrm{He}$ is currently an Associate Professor at University Pierre-et-Marie-Curie (UPMC), Paris, France.

His research interests include numerical methods for electromagnetic (scattering, antennas and microwave circuits), metasurface structures, field beam expansion methods, electromagnetic band-gap structures.

Dr. Casaletti was a co-recipient of the Best Poster Paper Award at the 3rd European Conference on Antennas and Propagation (EuCAP-2009), Berlin, Germany, and the recipient of an Honorable Mention for Antenna Theory at EuCAP-2010, Barcelona, Spain, and the Best Paper Award on Antenna Theory at EuCAP-2011, Rome, Italy. 


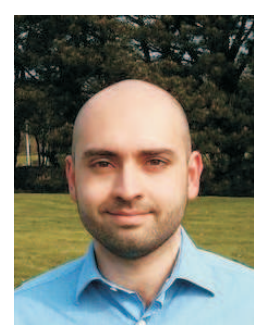

Guido Valerio (S'06-M'10) received the M.S. degree (cum laude and honorable mention) in electronic engineering in 2005, and the $\mathrm{Ph} . \mathrm{D}$. degree in applied electromagnetics in 2009, from La Sapienza, Rome, Italy. From February to August 2008 he was a Visiting Scholar at the University of Houston, TX, USA. In 2011, he joined the Institute d'Electronique et de Télécommunications de Rennes (IETR), France, where he is currently a researcher.

His scientific interests involve numerical methods for wave propagation and scattering in complex structures. He studied efficient computation and interpolation schemes for periodic Green's functions, the rigorous modeling of the interaction of nonperiodic sources with periodic media, modal properties of multilayered structures, full-wave methods for large SIW multilayered antennas. He was involved in the GPR characterization of Martian soil, and in the design of UWB antennas, harmonic-tunable active antennas, leaky-wave antennas, multifunction antenna arrays for weather and aircraft surveillance.

In 2008 Dr. Valerio was the recipient of the "Leopold B. Felsen Award for Excellence in Electrodynamics." In 2009 he was a finalist for the "Young Engineering Prize" at the European Microwave Conference. In 2010 he was the recipient of the "Barzilai Prize" for the best paper at the National Italian Congress of Electromagnetism (XVIII RiNEm).

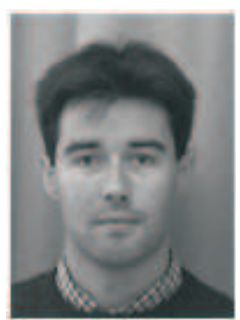

Ronan Sauleau (M'04-SM'06) graduated in electrical engineering and radio communications from the Institut National des Sciences Appliquées, Rennes, France, in 1995. He received the Agrégation degree from the Ecole Normale Supérieure de Cachan, France, in 1996, and the Doctoral degree in signal processing and telecommunications and the "Habilitation à Diriger des Recherches" degree from the University of Rennes 1, France, in 1999 and 2005, respectively.

He was an Assistant Professor and Associate Professor at the University of Rennes 1, between September 2000 and November 2005, and between December 2005 and October 2009, respectively. He has been appointed as a full Professor in the same University since November 2009. His current research fields are numerical modeling (mainly FDTD), millimeter-wave printed and reconfigurable (MEMS) antennas, substrate integrated waveguide antennas, lens-based focusing devices, periodic and non-periodic structures (electromagnetic bandgap materials, metamaterials, reflectarrays, and transmitarrays) and biological effects of millimeter waves. He has been involved in more than 30 research projects at the national and European levels and has co-supervised 14 post-doctoral fellows, $24 \mathrm{PhD}$ students and 40 master students.

He has received eight patents and is the author or coauthor of more than 140 journal papers and 300 publications in international conferences and workshops. He has shared the responsibility of the research activities on antennas at IETR in 2010 and 2011. He is now co-responsible for the research Department 'Antenna and Microwave Devices' at IETR and is deputy director of IETR. Prof. Sauleau received the 2004 ISAP Conference Young Researcher Scientist Fellowship (Japan) and the first Young Researcher Prize in Brittany, France, in 2001 for his research work on gain-enhanced FabryPerot antennas. In September 2007, he was elevated to Junior member of the "Institut Universitaire de France". He was awarded the Bronze medal by CNRS in 2008. He was the co-recipient of several international conference awards with some of his students (Int. Sch. of BioEM 2005, BEMS'2006, MRRS'2008, E-MRS'2011, BEMS'2011, IMS'2012, Antem'2012). He served as a guest editor for the IEEE Antennas Propogat. Special Issue on "Antennas and Propagation at $\mathrm{mm}$ and sub $\mathrm{mm}$ waves".

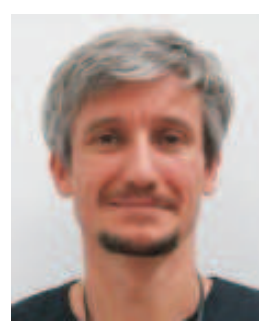

Laurent Le Coq received the electronic engineering and radiocommunications degree and the french DEA degree (M.Sc.) in electronics in 1995 and the Ph. D. in 1999 from the National Institute of Applied Science ( INSA), Rennes, France. In 1999, he joined IETR (Institute of Electronics and Telecommunications of Rennes), University of Rennes 1, as a research lab engineer, where he is responsible for measurement technical facilities up to $110 \mathrm{GHz}$.

His activities in antenna measurements and development of related procedures involved him in more than 30 research contracts of national or european interest. He is author and co-author of 35 journal papers and 35 papers in conference proceedings.

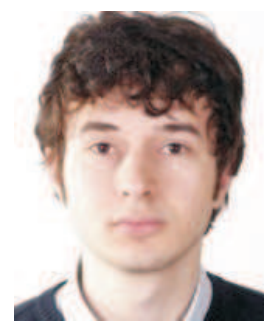

Santi Concetto Pavone was born in Patti (ME), Italy, in 1988. He received the Bachelor's Degree (B.Sc.) in Electronics Engineering (Cum Laude) in 2010 and the Master's Degree (M.Sc.) in Electronics Engineering (Cum Laude) in 2012, both from the University of Messina, Italy. During his Master's Thesis, he designed a Low Noise Amplifier and an Integrated Antenna for Millimeter Waves Applications $(60 \mathrm{GHz})$, using a CMOS SOI $65 \mathrm{~nm}$ Technology for Analog ICs. Currently, he is a Ph.D. Student in Information Engineering and Science (IES), research line in Electromagnetics Engineering, at the University of Siena, Italy. His research interests include leaky-wave antennas, focusing systems at microwaves, mw electronics and optoelectronics.

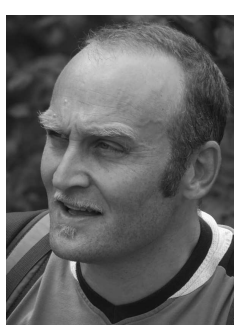

Matteo Albani (M'98 - SM'10) received the Laurea degree in Electronic Engineering (1994) and the Ph.D. degree in Telecommunications Engineering (1999) from the University of Florence, Italy.

$\mathrm{He}$ is currently an Adjunct Professor in the Information engineering Department, University of Siena, Italy, where he is also Director of the Applied Electromagnetics Lab. From 2001 to 2005 he was an Assistant Professor at the University of Messina, Italy.

His research interests are in the areas of highfrequency methods for electromagnetic scattering and propagation, numerical methods for array antennas, antenna analysis and design. He coauthored more than 40 journal papers and more than 140 conference papers.

Dr. Albani was awarded the "Giorgio Barzilai" prize for the Best Young Scientist paper at the Italian National Conference on Electromagnetics in 2002 (XIV RiNEm). 